\title{
EXCITED STATES IN SOLID PHENOTHIAZINE AS STUDIED BY ELECTROABSORPTION SPECTROSCOPY
}

\author{
J. KALINOWSKI ${ }^{a, b}$, W. STAMPOR ${ }^{b}$, V. FATTORI ${ }^{a}$ AND P. Di MARCo ${ }^{a}$ \\ ${ }^{a}$ Institute of Photochemistry and Radiation, C.N.R. \\ Via P. Gobetti 101, 40129 Bologna, Italy \\ ${ }^{b}$ Department of Molecular Physics, Technical University of Gdanisk \\ G. Narutowicza 11/12, 80-952 Gdanisk, Poland
}

(Received March 16, 1995)

Dedicated to Professors Krzysztof Pigoń, Józef W. Rohleder and Zdzisław Ruziewicz on the occasion of their 70lh birthday*

Electric field modulated absorption spectra of vapor deposited layers of phenothiazine, explained in terms of a quadratic Stark effect, indicate modification of intramolecular (Frenkel-type) excitons by charge transfer transitions throughout UVIS absorption spectrum in the range $250-450 \mathrm{~nm}$. The charge transfer character of the excited states appears at energies characteristic of various oxidation products of phenothiazine as identified from a spectroscopic study of photochemical air oxidation, performed on solution and solid film samples of this compound. The charge transfer transitions are ascribed to electron transfer between the molecules of phenothiazine and their oxidized species created in the samples during preparation and handling procedures.

PACS numbers: $31.50 .+w, 31.70 . D k, 31.70 . K s, 33.55 . B e$

\section{Introduction}

Phenothiazine (PHT) and its derivatives have received a great deal of attention since over the past 50 years [1]. Of particular interest have been their electronic excited states studied by UV absorption spectroscopy (see e.g. [2-4]). Quantum mechanical calculations [5-7], numerous examples of electrochemical and enzymathic oxidation [1] as well as formation of charge-transfer (CT) complexes with various acceptors [8-11] show the PHT ring to be a powerful electron donor.

*Submitted on invitation of the Insticute of Physical and Theoretical Chemistry, Technical University of Wrocław, Wrocław, Poland. 
These electronic properties have led PHT derivatives to various applications in technological processes (antioxidants) [12] and pharmacy (anthelmintic agents, psychotropic drugs) $[10,11,13]$.

The donor properties of molecular PHT show up in the solid state as fairly low-ionization potential (5:1 eV [14-16]) and can be expected to generate low-energy CT excitons. CT excitons in single-component molecular solids are of great interest because following their properties the primary charge separation steps can be studied. These, in turn, are of the fundamental importance in understanding of such phenomena as photocarrier generation in photoconductors, photo-redox reactions and photosynthesis. In fact, a CT transition has been suggested to appear outside of the strong local excitation region in the absorption spectrum of PHT crystal [17]. This suggestion is based on weak absorption bands [17] and principal photoconduction maximum $[17,18]$ above $400 \mathrm{~nm}$, supported by an electroabsorption [17] and two-color, two-step excited photocurrent [18] experiments on single crystals of PHT, performed in ambient atmosphere air. In contrast, steady-state photoconductivity studies on polycrystalline films of PHT under similar conditions show only a weak feature at about $410 \mathrm{~nm}$, suggesting lack or only minor role of low-energy CT states in photogeneration of charge [19]. On the other hand, a strong photocurrent response in the UV absorption region suggests high-energy excited states to be major contributors to charge separation processes. In order to verify this result and to search for CT excitons in solid PHT we have performed electroabsorption (EA) experiments in a broad spectral range $(250-450 \mathrm{~nm})$ on thin films allowing measurements at high electric fields. An additional spectroscopic study of photochemical air oxidation of PIT in solution and solid films has been done to support the suggestion on the crucial role of oxidation products for the shape of absorption and electroabsorption spectra of solid phenothiazine.

\section{Experimental details}

\subsection{Sample preparation}

Commercially available PHT $\left(\mathrm{C}_{12} \mathrm{H}_{9} \mathrm{NS}\right)$ was purified by multiple sublimation. Care was taken not to expose the material to the ambient atmosphere and light during purification process since PHT is known to form various photo-oxidized products [20]. However, transfer of the purified material to the evaporation quartz vessel was done in contact with ambient air. The films were prepared on room-temperature, carefully cleaned quartz substrates by evaporation under vacuum $\left(\approx 5 \times 10^{-3} \mathrm{~Pa}\right)$ at a high rate of $5 \mathrm{~nm} / \mathrm{s}$ and heating temperature $\approx 120^{\circ} \mathrm{C}$. Typically the film thicknesses were between 0.2 and $1 \mu \mathrm{m}$ as measured with a digital profilometer (Gimetr VIS model I). Such obtained polycrystalline films were contacted with partially transparent (5-30\% transmission in visible) $\mathrm{Al}$ electrodes in a sandwich-configuration arrangement, and mounted in an ambient-light protected environmental chamber. The Al contacts ensure low level of charge injection, allowing neglect of space-charge effects. Electromodulation experiments with a surface electrode configuration were made on the samples immersed in silicone oil to prevent breakdown along the surface. All measurements were done at room temperature. 


\subsection{Electroabsorption measurements}

The EA measurements were performed at the first $(1 \omega)$ and 2 nd $(2 \omega)$ harmonics of the fundamental frequency $(\omega)$ of a sinusoidal electric field, $F=F_{0} \sin (\omega t)$, applied to the illuminated electrode. The frequency of the applied electric field was varied between $100 \mathrm{~Hz}$ and $10 \mathrm{kHz}$, but no change in the EA signals has been observed. The ac modulation of light intensity $(I)$ passing through the sample with different dc biases could be detected. The experimental setup to record the EA signal, $\Delta I / I$, as a function of wavelength $(\lambda)$ is described elsewhere [21]. The spectral resolution was $\Delta \nu \approx 100 \mathrm{~cm}^{-1}$. The ordinary absorption spectra and their derivatives were recorded with a Zeiss spectrophotometer SPECORD-M42 and a Perkin-Elmer-Lambda 9 UVIS spectrophotometer. To measure excitation and luminescence spectra a Spex Fluorolog 2 spectrofluorometer was used.

\section{Results and discussion}

\subsection{Absorption and electroabsorption spectra}

The room-temperature ordinary absorption (OA) and electroabsorption (EA) spectra are shown for PHT in Fig. 1. The first (lower energy) absorption band is characterized by the strong maximum $332 \mathrm{~nm}$ in the deconvoluted Gaussian profiles shown in Fig. 1b. The best fit (dotted line) was obtained with four additional Gaussian profiles including a broad one (1), most probably, due to scattering of the light on the microcrystallites being distributed randomly in size and orientation. The above analysis followed a summation of Gaussian profiles: $D=\varepsilon d=\sum D_{n}$, where $\varepsilon=\left[\log \left(I_{0} / I\right)\right] / d, d$ is the thickness of the sample, $D_{n}=a_{n} \exp \left\{-\left[\left(E-E_{n}\right) / \sigma_{n}\right]^{2}\right\}, \sigma_{n}=W_{n} / 2(\ln 2)^{1 / 2}, W_{n}=$ FWHM, $E_{n}$ is the peak position, and $a_{n}$ - the amplitude for the $n$-th Gaussian. If the first absorption band at $332 \mathrm{~nm}$ represents $S_{0} \rightarrow S_{1} 0-0$ absorption transition, the second well-resolved band at $240 \mathrm{~nm}$ may be associated with the second singlet transition $S_{0} \rightarrow S_{2}$. Both are shifted with respect to their counterparts in solution, peaking at $320 \mathrm{~nm}$ and $254 \mathrm{~nm}$ (Fig. 1a). The bathochromic shift of the first band and hypsochromic shift of the second band can be associated with oxidation products present in the air- and light-exposed films. While the $240 \mathrm{~nm}$ maximum would reflect the presence of phenothiazone- 3 characterized by the absorption maximum at $237 \mathrm{~nm}$, the maximum at $332 \mathrm{~nm}$ shows a reasonable correspondence to the absorption band at $335 \mathrm{~nm}$ of phenothiazine-5-oxide (sulfoxide: PHT=O) (cf. Sec. 3.2). A support for this supposition comes from an additional feature in the film absorption that is from the broad shoulder located by the Gaussian profile 4 at $275 \mathrm{~nm}$ (Fig. 1b). This position corresponds well to characteristic maxima of phenothiazone-3 (270-287 $\mathrm{nm}$ ) and sulfoxide $(270 \mathrm{~nm})$ (see Sec. 3.2). Cationic and cationic radical species of PHT could contribute partly to this absorption band, but since their other characteristic bands between $420-455 \mathrm{~nm}$ [22] are not resolvable in the OA spectrum, this contribution should be of minor importance.

An interesting result comes from an observation that the principal minimum of the solution spectrum at $282 \mathrm{~nm}$ shifts to $302 \mathrm{~nm}$ in the absorption spectrum of the film. The latter corresponds strictly to one of the principal absorption maxima 

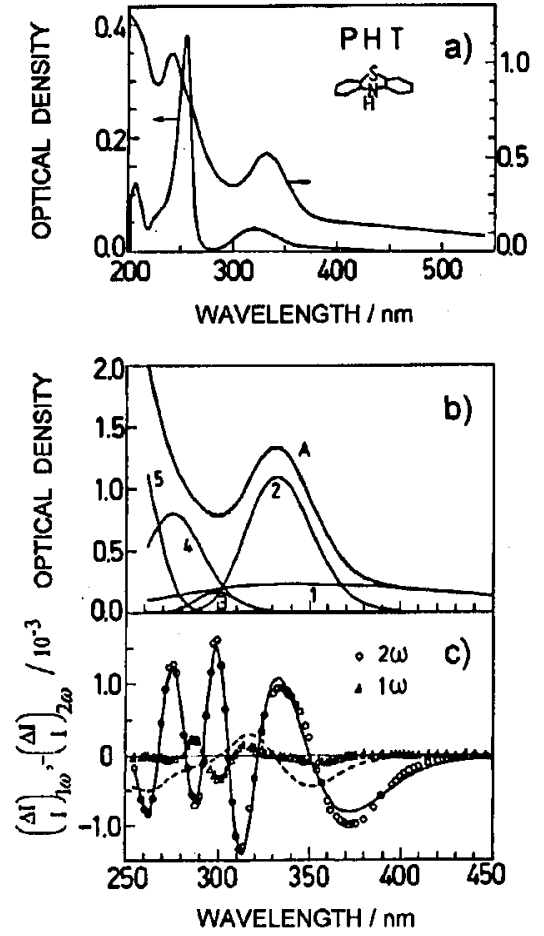

Fig. 1. The absorption spectrum ((a): right-hand scale) and its Gaussian profile analysis (b) of a $0.25 \mu \mathrm{m}$ thick PHT film at room temperature (solid line: experiment, dotted line: five band superposition of the Gaussian profiles). The absorption spectrum of a freshly prepared dilute solution $\left(10^{-5} \mathrm{M}\right)$ of PHT in ethanol is shown for comparison ((a): left-hand scale). Part (c): $1 \omega$ and $2 \omega$ electroabsorption spectra for the film sample, obtained with the electric field amplitude $F_{0}=8.3 \times 10^{5} \mathrm{~V} / \mathrm{cm}$ at room temperature (open circles and triangles: experimental results, solid line: best theoretical fit according to Eq. (2), dashed line: the first derivative term contribution according to Eq. (2) (cf. Table)).

of $\mathrm{PHT}=\mathrm{O}$ [22]. The strong peak at $300 \mathrm{~nm}$ in the second harmonic $(2 \omega)$ signal of the EA spectrum of the film is evidently associated with the small absorption maximum described by the Gaussian component 3 (Fig. 1b). To understand this strong EA response to a very little feature in the OA spectrum a brief analysis of the EA spectra is needed. The differential character of the $2 \omega$ EA curve (Fig. 1c) and quadratic increase in the $2 \omega$ EA signal with the applied voltage around the principal maximum and some other wavelengths (Fig. 2a) suggest interpretation of the EA spectra in terms of the Stark shift of electronic transition levels, caused by the interaction of molecular dipoles (permanent and induced) with local electric field (e.g. [21]). The EA signal can thus be expressed as

$$
\left(\frac{\Delta I}{I}\right)_{2 \omega}=\frac{\ln 10}{2 \sqrt{2}} F_{\mathrm{e}}^{2}\left[\frac{1}{2} \Delta p_{n} \frac{\mathrm{d} D_{n}}{\mathrm{~d} E}+\frac{1}{6}\left(\Delta \mu_{n}\right)^{2} \frac{\mathrm{d}^{2} D_{n}}{\mathrm{~d} E^{2}}\right],
$$



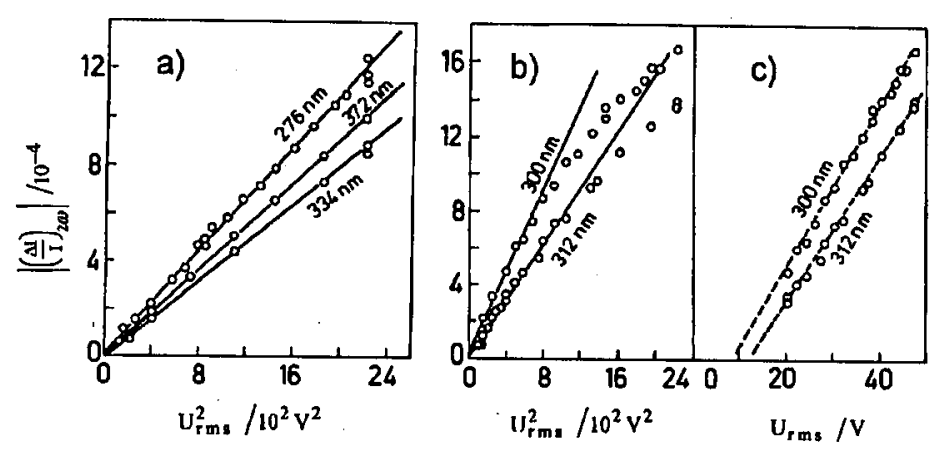

Fig. 2. The EA signal detected at the second harmonic versus the rms voltage $\left(U_{\mathrm{rms}}\right)$ applied to the sample $d=0.80 \mu \mathrm{m}$ at various wavelengths. Note the deflection from the quadratic behaviour of the signals shown in the plots versus the second (b) and first (c) power of $U_{\text {rms }}$.

where $\Delta p_{n}, \Delta \mu_{n}$ denote the average change of the polarizability, $p_{n}$, and permanent dipole moment, $\mu_{n}$, in $n$-th electronic transition characterized by the optical density $D_{n}$. The amplitude of the field-induced change of the $(\Delta I / I)_{2 \omega}$ is proportional to the square of the electric field acting on the molecule, the proportionality coefficient being a combination of the first and second derivatives of the absorption spectrum with respect to energy $E$. Formula (1) assumes a veraging over a totally random distribution of molecular dipoles. A good fit to the experimental $2 \omega$ EA spectrum is obtained on the basis of Eq. (1) (cf. Fig. 1c), assuming $\Delta p_{n}=\Delta p$ common for all electronic transitions approximated by 3 meaningful Gaussians displayed in Fig. 1b, and taking a sum over three different $\Delta \mu_{n}$ for the second term, that is

$$
\left(\frac{\Delta I}{I}\right)_{2 \omega}=- \text { const }\left(a \frac{\mathrm{d} D}{\mathrm{~d} E}+\sum_{n=2}^{4} b_{n} \frac{\mathrm{d}^{2} D_{n}}{\mathrm{~d} E^{2}}\right) .
$$

It is found that the contribution of the first derivative is relatively small (see dashed curve in Fig. 1c), giving $\Delta p=(16 \pm 4) \AA^{3}$, the value comparable with molecular polarizability of PHT in the ground state, $p \approx 24 \AA^{3}$ [23]. The energies $\left(E_{n}\right)$ and intensities $\left(b_{n}\right)$ of the second derivative contributions are listed in Table, along with those obtained from the resolution of the OA spectrum shown in Fig. 1b. The fitting values of FWIIM $\left(W_{n}\right)$ and $\Delta \mu_{n}$ resulted from $b_{n}$ and (1) for the three bands are also given in the table. An excellent agreement between $E_{n}$ obtained from OA and EA spectra is readily seen. This supports our correct interpretation of the EA spectra and allows to discuss the obtained values of $\Delta \mu_{n}$. All of them are much greater than the difference $\Delta \mu=\mu_{\text {exc }}-\mu_{\mathrm{g}} \approx 1 \mathrm{D}$ resulting from the dipole moments of the ground, $\mu_{\mathrm{g}}=2.15 \mathrm{D}$ [24], and excited, $\mu_{\mathrm{exc}}=3.1 \mathrm{D}[25,26]$, state molecules of PHT. We suppose that the main reason for that is the presence of oxidation products of PIIT, particularly phenothiazine-5-oxide, which can act as acceptors leading to intermolecular electron transfer and formation of CT states. The difference in $\Delta \mu_{n}$ would reflect the degree of electron transfer and/or differences in local intermolecular distances if different products were involved in CT 
TABLE:

Results of the band resolution of the second derivative contribution to the EA spectrum of PHT, along with $E_{n}, W_{n}$ and $a_{n}$ obtained from the resolution of the OA spectrum.

\begin{tabular}{c|c|c|c|c|c|c|c}
\hline \hline \multirow{2}{*}{$\begin{array}{c}\text { Band } \\
\#\end{array}$} & \multicolumn{3}{|c|}{ OA spectrum } & \multicolumn{3}{c|}{ EA spectrum } & \multirow{2}{*}{$\Delta \mu_{n}[\mathrm{D}]$} \\
\cline { 2 - 6 } & $E_{n}\left[\mathrm{kK}^{*}\right]$ & $W_{n}[\mathrm{kK}]$ & $a_{n}$ & $E_{n}[\mathrm{kK}]$ & $W_{n}[\mathrm{kK}]$ & $b_{n}[\mathrm{a} . \mathrm{u}]$. & \\
\hline 2 & 30.14 & 3.82 & 1.09 & 30.14 & 4.58 & 62.3 & 7.2 \\
& $(332 \mathrm{~nm})$ & & & $(332 \mathrm{~nm})$ & & & \\
3 & 33.44 & 3.05 & 0.17 & 33.35 & 2.35 & 27.9 & 12.1 \\
& $(299 \mathrm{~nm})$ & & & $(300 \mathrm{~nm})$ & & & \\
4 & 36.34 & 4.80 & 0.80 & 36.04 & 3.50 & 83.5 & 10.2 \\
\hline
\end{tabular}

${ }^{*} 1 \mathrm{kK}=10^{3} \mathrm{~cm}^{-1}$

formation upon excitation. Action of the molecular oxygen diffused into the sample cannot be completely excluded since formation of the CT complexes between PHT and oxygen has been suggested [27]. The presence of oxygen is known to inhibit CT transitions between 10-methylphenothiazine and strong acceptors in acetonitrile, but unsubstituted phenothiazine showed no charge transfer transitions under any conditions in the same solvent [10]. Could it mean that a particular local crystal structure is a necessary condition to enable intermolecular electron transfer? A substantial modification of van der Waals intermolecular forces by the hydrogen bonding between strongly polarized $\mathrm{S} \rightarrow \mathrm{O}$ bond of $\mathrm{PHT}=\mathrm{O}$ and $\mathrm{N}-\mathrm{H}$ hydrogen atoms of PHT is a conceivable way to create such a structure. We have recently shown the intermolecular hydrogen bonding to induce low-energy CT excitons through formation of a quasi-one-dimensional molecular stacks in the $\gamma$ form of linear trans-quinacridone crystal [28]. In contrast, they are not observed in our present EA experiment with PHT (Fig. 1c). This contradicts the results obtained by Takase and Kotani [17] on single PHT crystals kept under similar conditions. A huge structured EA signal $\left(\approx 10^{-2}\right)$ above $400 \mathrm{~nm}$ has been observed by those authors in the surface electrode geometry of the crystal sample immersed in silicone oil. One could argue that as the low-energy CT states in single PHT crystal involve electron transfer in one of the cleavage plane directions, they cannot show up in our EA experiment since due to hypothetical high film order the applied field is perpendicular to this direction (cf. related problem for tetracene [29]). In order to check this argument we have performed a surface electrode configuration experiment with a solid film immersed in silicone oil, and the result is shown in Fig. 3. As in the sandwich geometry (Fig. 1c), the EA signal above $400 \mathrm{~nm}$ is very small and does not show any structure which could be associated with strong CT transitions. Its shape determined by the long-wavelength tail of the principal transition at $332 \mathrm{~nm}$ and most probably deformed by light scattering effects (represented by the first Gaussian component in Fig. 1b) effectively masks weak CT transitions if there are any in this spectral region. In conclusion, the crystallite orientation effect as a reason for the lack of a meaningful EA signal structure above 


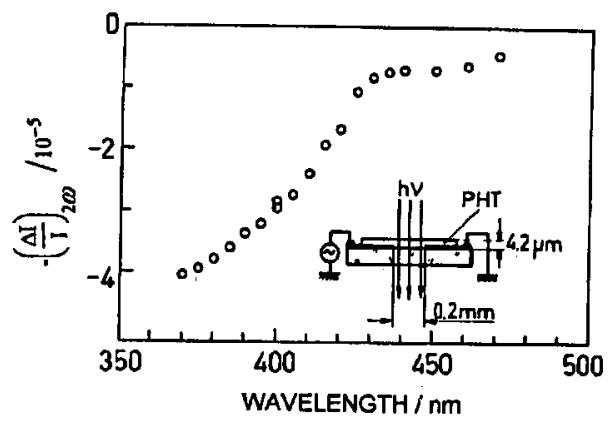

Fig. 3. The $2 \omega$ EA spectrum in the long-wavelength part of the absorption spectrum measured in the surface electrode configuration as shown in the insert. The field amplitude $F_{0}=5.7 \times 10^{4} \mathrm{~V} / \mathrm{cm}$.

$400 \mathrm{~nm}$ can be ruled out. Therefore, the origin of the two absorption bands in this spectral range (415 and $430 \mathrm{~nm}$ ) observed at $15 \mathrm{~K}$ [17] is still an open question. Interestingly, principal emission maxima for both PHT in solution and solid films at room temperature ( 420 and $445 \mathrm{~nm}$ ) appear close to these bands (see Sec. 3.2).

On the contrary, the CT nature of the states below $400 \mathrm{~nm}$ seems to be well supported by notable deflection in the quadratic dependence of $(\Delta I / I)_{2 \omega}$ versus $U_{\text {rms }}$ (Fig. 2b, c) and $1 \omega$ EA signal which shows maximum of the absolute value around $300 \mathrm{~nm}$ (Fig. 1c). These features can be explained by the presence of an internal electric field resulting from the inhomogeneously distributed space charge generated efficiently at this wavelength as comes also from photoconduction studies [19] (a discussion of internal fields seen through EA measurements is given in Ref. [30]). In other words, an efficient charge separation process is active within this absorption band. Moreover, the most pronounced peak at $570 \mathrm{~nm}$ in the two-photon-induced photocurrent spectrum [31] falls in the high one-photon photoconduction region between 277 and $300 \mathrm{~nm}$ [19], where strong EA features are coupled with big changes in the dipole moment (see Table).

\subsection{Photochemical air oxidation}

A crucial role ascribed to PHT oxidation products in absorption and electroabsorption behaviour of solid films of PHT imposed a strong demand of a spectroscopic photochemical air oxidation study. In our present study, solution and solid film samples were exposed to the selected UV light from a Xenon $900 \mathrm{~W}$ lamp or to ordinary indoor light in the ambient atmospheric air. Successive absorption spectra of the irradiated and non-irradiated samples were recorded and subtracted, giving a differential transmission which allows the observation of changes in the spectrum. Such differential spectra are shown in Fig. 4. The absorption peaks at $230,270-288,370$ and $495 \mathrm{~nm}$ for the solution spectra correspond well to major absorption maxima of phenothiazone-3 [22], that is to a photo-oxidation product of PHT obtained, among others, from irradiated ethanol solutions in photochemical studies [20]. They are absent in the absorption of the irradiated PHT film. Instead, the maximum at $313 \mathrm{~nm}$ can be related to the maximum at $310 \mathrm{~nm}$ observed in absorption spectra of solutions of phenothiazine-5-oxide $(\mathrm{PHT}=\mathrm{O})$ in $\mathrm{CCl}_{4}$ [22]. 

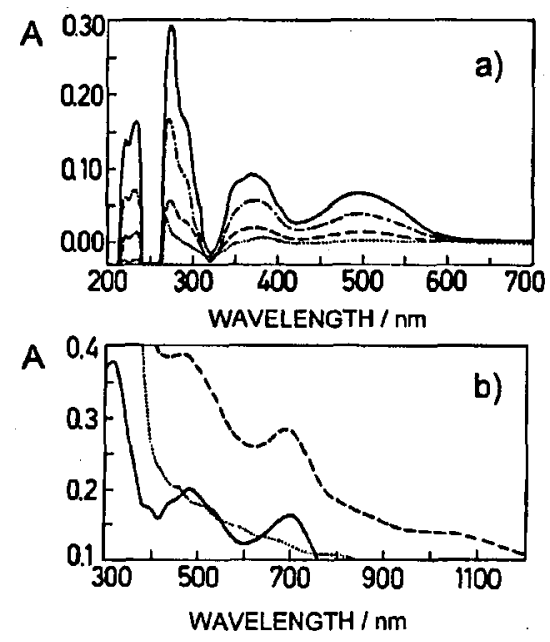

Fig. 4. Absorption spectra of photo-oxidized products of PHT: (a) in ethanol solution $\left(10^{-4} \mathrm{M}\right)$ after periods of $30(\cdots), 90(-\cdots), 240(\cdots \cdots)$ and $520 \mathrm{~min}(-)$ of irradiation with UV light. (b) $1 \mu \mathrm{m}$ thick film after a $24 \mathrm{~h}$ irradiation period (solid line): dotted and dashed lines are the absorption spectra of the film before and after irradiation, respectively; the solid line is obtained by subtracting of these two spectra. In both cases ((a) and (b)) a Xenon $900 \mathrm{~W}$ lamp light flux of $10^{14}$ quanta $/ \mathrm{cm}^{2} \mathrm{~s}$ at $330 \mathrm{~nm}$ was used for irradiation.

IIowever, the second major peak at $480 \mathrm{~nm}$ and minor features at 395 and $534 \mathrm{~nm}$ can be again assigned to phenothiazone- 3 as it is known to shift the absorption maxima with change in solvents $[20,32]$. A weak shoulder at $437 \mathrm{~nm}$ and meaningful maximum at $696 \mathrm{~nm}$ are indicative of some PHT ionic species: the cation PHT radical (PIIT ${ }^{+}$) and dication PIT $^{2+}$ [22]. It is clear from the above that oxidation products appear in irradiated PHT samples exposed to atmospheric air, even so their distribution in solution and solid state are different. While in solution the major absorption features belong to phenothiazone-3, in solid films PHT=O and cationic species are mostly detected. This would agree with conclusions drawn previously from the EA experiment (cf. Sec. 3.1).

Emission spectra also show some evalution with irradiation, but location of their most characteristic features remains unchanged (Fig. 5). The fluorescence spectrum of PHT in dilute solution $\left(10^{-5} \mathrm{M}\right)$ seems to show a weak structure around $310 \mathrm{~nm}$ possibly corresponding to the $\mathrm{O}-\mathrm{O}$ transition in absorption (Fig. 1a). Further structures at energies lower successively by $0.40,0.27,0.23,0.17$ and $0.20 \mathrm{eV}$ do not have their counterparts in the first absorption band system. Positions and heights of the maxima at $420,445 \mathrm{~nm}$, and broad shoulder at $\approx 480 \mathrm{~nm}$ do not change with concentration so that excimer formation must be rather excluded. Clearly, the emission spectrum of PHT in solution should not be considered as the mirror image of its first absorption band. Solution samples exposed to light exhibit increasing emission close to $480 \mathrm{~nm}$ as irradiation time increases (Fig. $5 \mathrm{~b}$ ). Also, a long-wavelength maximum at about $620 \mathrm{~nm}$ increases with irradiation 

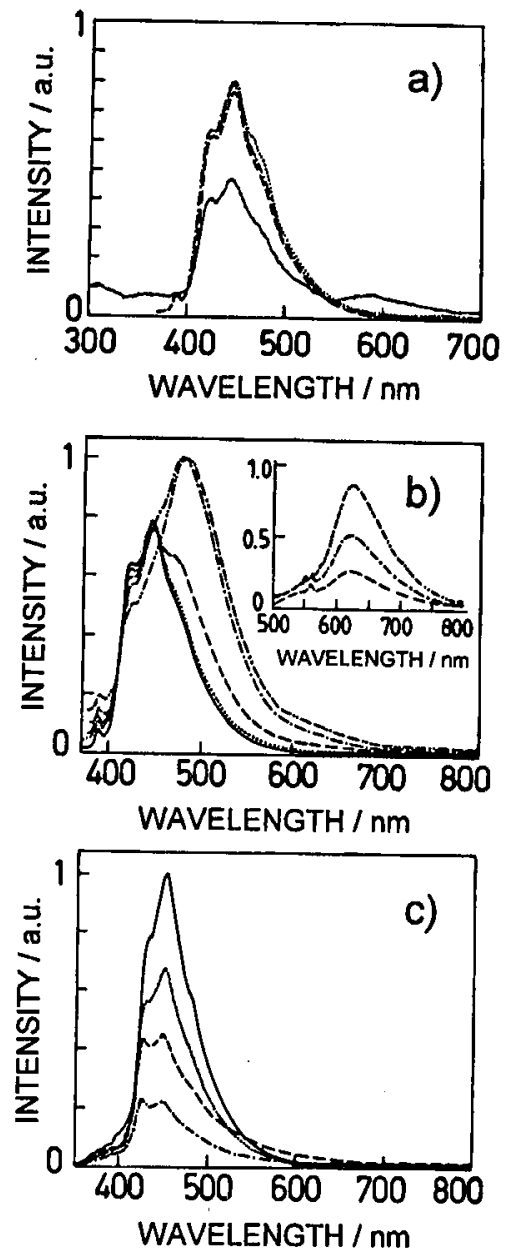

Fig. 5. Emission spectra of freshly prepared and UV photo-oxidized samples of PHT: (a) freshly prepared ethanol solutions at different concentrations of $10^{-5}(-), 10^{-4}$ $(-\cdots), 10^{-3}(-\cdots)$ and $10^{-2} \mathrm{M}(\cdots)$ ) of PHT; excitation wavelengths have been adjusted to the optical density of the samples, starting from $260 \mathrm{~nm}$ at $10^{-5} \mathrm{M}$, through 350 at $10^{-4} \mathrm{M}$, and $385 \mathrm{~nm}$ at $10^{-3} \mathrm{M}$, up to $410 \mathrm{~nm}$ at $10^{-2} \mathrm{M}$; (b) in ethanol solution $\left(10^{-4} \mathrm{M}\right)$ after irradiation periods of $0(-), 30(\cdots), 90(---)$, $240(-\cdot)$, and $520 \mathrm{~min}(-\ldots .-)$; excitation wavelength $\lambda_{\text {exc }}=350 \mathrm{~nm} ; \mathrm{a}$ long-wavelength part of the spectrum excited at $\lambda_{\text {exc }}=480 \mathrm{~nm}$ is shown in the inset; (c) $1 \mu \mathrm{m}$ thick film after irradiation periods $\left.0(-), 360 \mathrm{~min}(\cdots \cdots), 15(--)^{-}\right)$ and $24 \mathrm{~h}(-\cdots) ; \lambda_{\text {exc }}=330 \mathrm{~nm}$. Irradiation conditions as described in Fig. 4.

time. This would suggest these emission bands to have their origin in the oxidation products. They correspond to absorption bands of phenothiazone-3 (broad maximum at $495 \mathrm{~nm}$ in Fig. 4a) and dication PHT ${ }^{2+}(625 \mathrm{~nm}$ [22]). As could be predicted from a differeit distribution of oxidation products, these emission bands 
are of much less importance in solid films (Fig. 5c). The positions of two major peaks at 420 and $445 \mathrm{~nm}$ also present in the solution spectra do not change with irradiation of the samples. The irradiation quenching of the total luminescence is accompanied by redistribution of intensity between these two maxima: a decrease in the fluorescence at 445 shows up as an increase in the peak intensity at $420 \mathrm{~nm}$. It is interesting to note that the peak at $420 \mathrm{~nm}$ corresponds to a characteristic absorption maximum of ionic species $\left(\mathrm{C}_{12} \mathrm{H}_{8} \mathrm{NS}\right)^{+}$and that at $445 \mathrm{~nm}$ to ionic species $\left(\mathrm{C}_{12} \mathrm{H}_{8} \mathrm{NS}-\mathrm{OH}\right)^{+}$(the species denoted as V and IX in Table I of Ref. [22]). These two species seem to be characteristic of a solid PHT as can be inferred from a comparison of emission and excitation fluorescence spectra shown in Fig. 6.

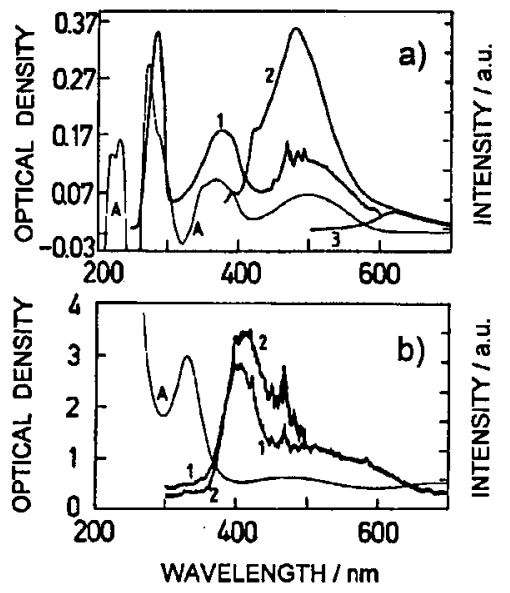

Fig. 6. Excitation fluorescence and emission spectra of photo-oxidized PHT: (a) in ethanol solution $\left(10^{-4} \mathrm{M}\right)$ after the irradiation period of $520 \mathrm{~min}$ (1: excitation spectrum recorded at the emission wavelength $\lambda_{\mathrm{em}}=620 \mathrm{~nm}, 2$ : emission spectrum at $\lambda_{\mathrm{exc}}=$ $350 \mathrm{~nm}, 3$ : emission spectrum at $\lambda_{\text {exc }}=480 \mathrm{~nm}$, absorption spectrum (A) against the non-exposed sample shown for comparison). (b) $1 \mu \mathrm{m}$ thick film exposed to indoor light and atmospheric air for 60 days (1: excitation spectrum recorded at $\lambda_{\mathrm{em}}=575 \mathrm{~nm}$, 2: emission spectrum at $\lambda_{\text {exc }}=240 \mathrm{~nm}$, absorption spectrum (A) shown for comparison).

Though in both solution and solid film samples excitation fluorescence spectra are mapping principal emission features, the difference comes from their origin as identified above. In solution samples (Fig. 6a) they belong to the ionic species of lower oxidation level than those in solid film samples (Fig. 6b). On the basis of the above observations it is suggested that ionic species $\left(\mathrm{C}_{12} \mathrm{H}_{8} \mathrm{NS}\right)^{+}$and $\left(\mathrm{C}_{12} \mathrm{H}_{8} \mathrm{NS}-\mathrm{OH}\right)^{+}$ are produced in the preparation procedures of thin films as well as single crystals. They are responsible for emission and EA spectra above $400 \mathrm{~nm}$ leading to CT transitions in combination with neutral molecules of PIT. The difference in the $2 \omega$ EA response for thin films and thick crystals arises from different contributions of the second derivative factors in Eqs. (1) and (2), a consequence of the difference in optical densities of these two types of samples. 


\section{Conclusions}

Whereas CT transitions are dominant features in the ordinary optical absorption spectra of donor-acceptor complexes in the visible [33], their presence in single component molecular solids can be successfully monitored by electroabsorption measurements. The usefulness of the electromodulation technique has been demonstrated in resolving a CT transition at energies around and below the intramolecular (Frenkel type) transitions of the first absorption bands in linear trans-quinacridone polycrystalline layers [28] and phenothiazine single crystals [17], respectively. In the latter case it has been originally assigned to charge-transfer excitons formed by electron transfer between phenothiazine molecules [17].

The present results show that charge transfer transitions appear throughout entire UVIS absorption spectrum attainable in the experiment on solid state phenothiazine, having their origin in electron transfer from phenothiazine molecules to their various oxidation products formed in the course of preparation and handling the samples. By this result the existence of CT excitons in the absorption edge (below the first Frenkel-type transition) in single-component molecular solids becomes again subject of controversy and requires further studies.

\section{References}

[1] C. Bodea, I. Silberg, in: Advances in Heterocyclic Chemistry, Eds. A.R. Katrizky, A.J. Boulton, Vol. 9, Academic Press, New York 1968, p. 321.

[2] J.A. Van Allan, G.A. Reynolds, R.E. Adel, J. Org. Chem. 27, 1659 (1962).

[3] S. Saraf, Heterocycles 19, 935 (1982).

[4] M.B. Ryzhikov, A.N. Rodionov, A.N. Stiepanov, Zh. Fiz. Khim. 63, 2515 (1989).

[5] M.K. Orloff, D.D. Fitts, Biochim. Biophys. Acta 47, 596 (1961).

[6] J.P. Marlieu, B. Pullman, Theor. Chim. Acta 2, 293 (1964).

[7] A. Mehlhorn, B. Schwenzer, K. Schwetlick, Tetrahedron 33, 1483 (1977).

[8] M. Kinoshita, Bull. Chem. Soc. (Jpn.) 35, 1609 (1962).

[9] R. Beukers, A. Szent-Gyórgyi, Recueil Trav. Chim. 81, 255 (1962).

[10] R. Foster, P. Hanson, Biochim. Biophys. Acta 112, 482 (1966).

[11] R. Foster, C.A. Fyfe, Biochim. Biophys. Acta 112, 490 (1966).

[12] T. Colclough, J.I. Cunneen, J. Chem. Soc. , 4791 (1964).

[13] G. Karreman, I. Isenberg, A. Szent-Gyórgyi, Science 130, 1191 (1959).

[14] L.E. Lyons, J.C. Mackie, Nature 197, 589 (1963).

[15] N. Sato, H. Inokuchi, B.M. Schmid, N. Karl, J. Chem. Phys. 83, 543 (1985).

[16] I. Seki, Mol. Cryst. Liq. Cryst. 171, 255 (1989).

[17] T. Takase, M. Kotani, J. Chem. Phys. 90, 2134 (1989).

[18] A. Kurabayashi, M. Kotani, Mol. Cryst. Liq. Cryst. 183, 193 (1990).

[19] G. Giro, J. Kalinowski, P. Di Marco, V. Fattori, in: Proc. 6th Int. Conf. Electr. \& Related Properties of Organic Sol., Ed. J. Kalinowski, Gordon and Breach, Philadelphia 1993, p. 207.

[20] G.P. Brown, J.W. Cole, T.I. Crowell, J. Org. Chem. 20, 1772 (1955). 
[21] W. Stampor, J. Kalinowski, P. Di Marco, Chem. Phys. 134, 385 (1989).

[22] II.J. Shine, E.E. Mach, J. Org. Chem. 30, 2130 (1965).

[23] V.E. Umanskii, A.V. Luzanov, I.V. Krivoshei, Zh. Strukt. Khim. 15, 1024 (1974).

[24] A.L. Mc Clellan, Tables of Experimental Dipole Moments, W.H. Freeman, San Francisco 1963.

[25] A.T. Amos, B.L. Burrows, Adv. Quant. Chem. 7, 303 (1973).

[26] D. Grasso, E. Bellio, Chem. Phys. Lett. 30, 421 (1975).

[27] HI.F. Martin, S. Price, B.J. Gudzinowicz, Archiv. Biochem. Biophys. 103, 196 (1963).

[28] J. Kalinowski, W. Stampor, P. Di Marco, Chem. Phys. 182, 341 (1994).

[29] J. Kalinowski, W. Stampor, B. Petelenz, P. Petelenz, Chem. Phys. 167, 185 (1992).

[30] W. Stampor, J. Kalinowski, P. Di Marco, in Ref. [19], p. 233.

[31] A. Miniewicz, A Samoć, D.F. Williams, Mater. Sci. 10, 185 (1984).

[32] D.F. Houston, E.B. Kester, F. DeEds, J. Am. Chem. Soc. 71, 3816 (1949).

[33] D. Haarer, J. Chem. Phys. 67, 4076 (1977). 